\title{
RESEARCH
}

Open Access

\section{Cardiac repair in a murine model of myocardial infarction with human induced pluripotent stem cell-derived cardiomyocytes}

Xin Jiang ${ }^{1}$, Ziyi Yang ${ }^{2}$ and Ming Dong ${ }^{2 *}$

\begin{abstract}
Background: Cellular replacement strategies using human induced pluripotent stem cells (iPSCs) and their cardiac derivatives are emerging as novel treatments for post-myocardial infarction (MI) heart failure (HF); however, the mechanism of recovery of heart function is not very clear. The purpose of this study was to investigate the efficiency of using highly purified human induced pluripotent stem cell-derived cardiomyocytes (iPS-CMs) for myocardial repair in a mouse model of $\mathrm{Ml}$ and to clarify the mechanism of recovery of heart function.

Methods: Animals modelling Ml were randomly assigned to receive direct intramyocardial injection of culture medium (MI group) or $4 \times 10^{5}$ iPS-CMs (cell group) at the infarct border zone. Left ventricle (LV) performance was assessed with serial cardiac electrophysiology and was measured 1, 2 and 4 weeks post-MI. Invasive LV pressure measurement was measured at 4 weeks and was followed by sacrifice for histological examination.

Results: Compared to the Ml group, the left ventricle ejection fraction (LVEF), left ventricular internal diameter in end-diastole (LVIDd) and end-systole (LVIDs) and maximal positive and negative pressure derivative $( \pm d P / d t)$ were significantly improved in the iPS-CM group at 4 weeks post-MI. Histological examination revealed a very limited number of iPS-CMs 4 weeks after transplantation. Nonetheless, there was a significant enhancement of angiogenesis and a reduction in apoptosis of native cardiomyocyte after iPS-CM transplantation.
\end{abstract}

Conclusions: Our results demonstrate that transplantation of human iPS-CMs can improve heart function via paracrine action in a mouse model of myocardial infarction.

Keywords: Induced pluripotent stem cells, Cardiomyocytes, Myocardial infarction

\footnotetext{
*Correspondence: DongMing2020@hotmail.com

²Bioisland Laboratory, Biomedical Equipment Department, Building 3, No.188

KaiYuan Road, Huangpu District, Guangzhou, Guangdong, China

Full list of author information is available at the end of the article
}

C C The Author(s). 2020 Open Access This article is licensed under a Creative Commons Attribution 4.0 International License, which permits use, sharing, adaptation, distribution and reproduction in any medium or format, as long as you give appropriate credit to the original author(s) and the source, provide a link to the Creative Commons licence, and indicate if changes were made. The images or other third party material in this article are included in the article's Creative Commons licence, unless indicated otherwise in a credit line to the material. If material is not included in the article's Creative Commons licence and your intended use is not permitted by statutory regulation or exceeds the permitted use, you will need to obtain permission directly from the copyright holder. To view a copy of this licence, visit http://creativecommons.org/licenses/by/4.0/ The Creative Commons Public Domain Dedication waiver (http://creativecommons.org/publicdomain/zero/1.0/) applies to the data made available in this article, unless otherwise stated in a credit line to the data. 


\section{Background}

Myocardial infarction (MI) is a disease with high global mortality and morbidity rates. Permanent loss of cardiomyocytes (CMs) during MI contributes to progressive pathological left ventricular (LV) remodelling and heart failure (HF). Cell-based therapies using embryonic stem cells (ESCs) or induced pluripotent stem cells (iPSCs) and their cardiac derivatives have been proposed as an effective therapeutic approach to improve cardiac function in post-MI HF. ESCs and iPSCs are pluripotent stem cells that possess the ability to produce a sufficient amount of functional cardiomyocytes for "true" heart regeneration. Unlike ESCs, iPSCs are generated from an individual's own somatic cells. Thus, iPSC isolation has fewer ethical concerns, and immunosuppression is not needed after transplantation. iPSCs might overcome some of the limitations of human ESCs for heart regeneration. Indeed, iPSCs can be differentiated into functional cardiomyocytes both in vitro and in vivo [1, 2]. A few papers have reported the functional benefit of using these iPS-derived cardiomyocytes (iPS-CMs) in transplantation for heart regeneration in rat or pig MI models. After transplantation, iPS-CMs were able to actively integrate with the host myocardium and improve LV function [3-6]. However, iPSC differentiation is in the initial stage, and the mechanism by which these cells improve heart function is not very clear. In this paper, we investigate the efficiency of using highly purified iPS-CMs for myocardial repair in a mouse model of MI and hope to clarify the mechanism of heart function improvement.

\section{Methods}

\section{iPS-CM preparation}

Human iPS-CMs were purchased from Cellular Dynamics International (CMC-100-010-001, CDI, USA); $>4.0 \times 10^{6}$ viable cells were provided in cryovials. iCell cardiomyocytes labelled with green fluorescent protein (GFP), also from CDI, are highly purified human cardiomyocytes derived from iPSCs ( $>90 \%$ ) according to CDI's proprietary differentiation and purification protocols. Cells were shipped as cryopreserved suspensions of dissociated cells with iCell Cardiomyocytes Plating Medium and iCell Cardiomyocytes Maintenance Media. iCell cardiomyocytes were maintained in liquid nitrogen until further use to ensure optimal performance. Before injection, cells were thawed and suspended in culture medium at a concentration of $4 \times 10^{5} / 300 \mu \mathrm{l}$. iPS-CMs were characterised via the expression of the cardiac special marker troponin-T (1:200, Santa Cruz, CA). Cardiomyocytes were also assessed for senescence before transplantation with a $\beta$-Galactosidase Staining Kit (Cell Signaling Technology, \#9860) according to the manufacturer's instructions.

\section{Mouse model of $\mathrm{MI}$ and cell transplantation}

All animal experimental procedures were approved by the Committee on the Use of Live Animals in Teaching and Research at the University of Shenzhen. Adult male Imprinting Control Region (ICR) mice (12-16 weeks) underwent left anterior descending (LAD) coronary artery ligation to induce MI, as described previously [7]. Briefly, animals were anaesthetized with an intraperitoneal injection of ketamine $100 \mathrm{mg} / \mathrm{kg}$ and xylazine 20 $\mathrm{mg} / \mathrm{kg}$ and then were connected to a mouse ventilator via tracheal intubation. Acute myocardial infarction was induced by LAD ligation in the middle of the left side by 8-0 sutures introduced via left thoracotomy. A successful MI model was confirmed by myocardial blanching at the apex. Ten minutes after ligation, animals were randomised to receive direct intramyocardial injections of $30 \mu \mathrm{l}$ of (1) culture medium (MI group, $n=12$ ) or (2) $4 \times 10^{5}$ iPS-CMs (cell group, $n=12$ ) at 3 different LV sites near the infarct border area. Another group of mice $(n=10)$ that served as controls underwent thoracotomy without coronary artery ligation (control group). Different groups in the study were subjected to invasive haemodynamic assessment 4 weeks post-MI and then were sacrificed for histological evaluation. The experimental design and protocols performed in the study are summarised in Fig. 1.

\section{Heart function evaluation by echocardiography}

Echocardiography evaluations were performed using a Vevo 2100 high-resolution in vivo microimaging system equipped with a real-time microvisualisation scan head of $17.5 \mathrm{MHz}$ (VisualSonics, Toronto, Ontario, Canada). An experienced operator who was blinded to the animal model and treatment performed all echocardiography. In brief, mice were anaesthetized via intraperitoneal injection of ketamine and xylazine as described above, and then the left lateral decubitus position on the anterior chest wall was examined. Standard M-mode parameters, including left ventricular internal diameter in enddiastole (LVIDd) and end-systole (LVIDs), posterior wall thickness (LVPW) and intraventricular septum thickness (IVS), were measured to calculate left ventricular ejection function (LVEF) according to the American Society of Echocardiography recommendations [8]. The mean value of three different cardiac cycles was used for each individual mouse at each time point. Serial echocardiography was performed to assess LV function on days 7 , 14 and 28 after transplantation.

\section{Invasive cardiac haemodynamic assessment}

Before sacrifice, mice were anaesthetized and mechanically ventilated as described above. A 1.4-Fr Millar catheter was inserted into the LV cavity via the right carotid artery and then was connected to a pressure transducer. 


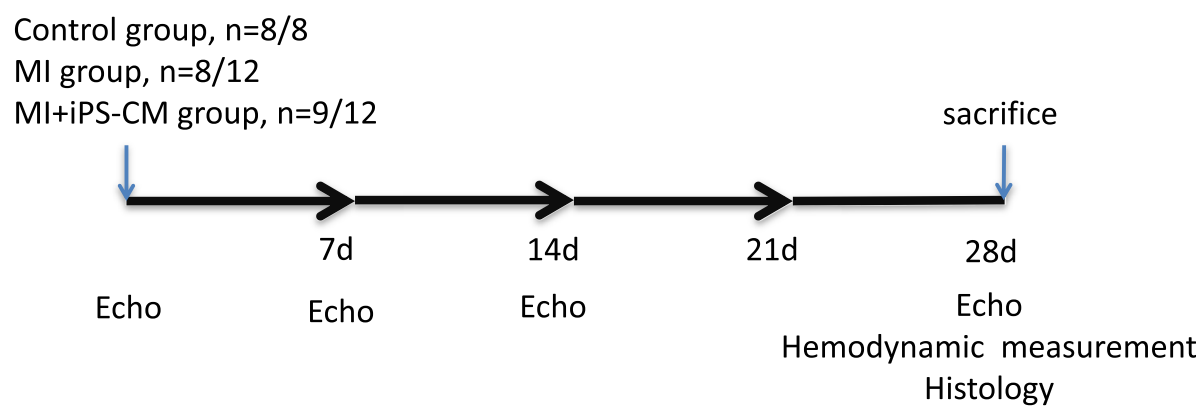

Fig. 1 Experimental design and protocols. Four mice in the Ml group and 3 mice in the iPS-CM group died during 4 weeks of observation after $\mathrm{Ml}$ induction. At least 8 animals in each group survived for the duration of the experiment. There was no significant difference in mortality rate between these two groups

Haemodynamic parameters, including maximal positive and negative pressure derivative $(\mathrm{dP} / \mathrm{dt}), \mathrm{LV}$ enddiastolic pressure (LVEDP) and LV end-systolic pressure (LVESP), were recorded with a PowerLab system (AD Instruments, Inc., Colorado Springs, CO).

\section{Histological evaluation}

Hearts were harvested from animals 4 weeks after MI. The mRNA levels of PAI-1, MMP-3 and IL- 6 were evaluated by RT-PCR to assess senescence in the control, MI and MI+ iPS-CM groups. Primer sequences for those mRNAs were as follows: PAI-1: 5' primer- GACACCCTCAGCAT GTTCATC and 3' primer-AGGGTTGCACTAAAC ATGTCAG; MMP-3: 5' primer-TTTAAAGGAAATCA GTTCTGGGCTATA and 3' primer-CGATCTTCTT CACGGTTGCA; and IL-6: 5' primer-ACACATGTTC TCTGGG AAATCGT and 3' primer-AAGTGCATCA TCGTTGTTCATACA. Other tissues were fixed with $10 \%$ buffered formalin and embedded in paraffin. Then, paraffin blocks were sectioned into 5 - $\mu$ m slides for haematoxylin and eosin (H\&E) staining and immunofluorescence and immunohistochemical evaluation. The size of the infarct area was analysed using a Masson Trichrome Stain Kit (\#HT15, Sigma). To assess the survival of engrafted cells and cardiac cell differentiation, cells were immunofluorescently labelled to detect GFP (green) and cardiac troponin-T (red) (1:200; Lab Vision, Fremont, CA) with double staining in tissues, and then they were visualised under a fluorescence microscopy. Staining with polyclonal rabbit anti-von Willebrand factor (vWF; AB7356, 1:200, Chemicon, Rosemont, IL) was used to measure capillary density in the infarct border area after cell transplantation. An in situ apoptotic cell death detection kit, POD (Roche Applied Science, Mannheim, Germany), which is based on the terminal deoxynucleotidyl nick end-labelling (TUNEL) assay, was used to evaluate apoptosis after cell transplantation. Quantitative analysis of positive vessels and apoptotic nuclei was performed in different sections at nine random fields from the infarct area and infarct border area in each animal. Each sample slice was photographed under a microscope (Olympus BX51, Olympus, Tokyo, Japan).

\section{Statistical analysis}

Continuous variables are expressed as the mean \pm SEM. A paired sample $t$ test was used to compare 2 groups, and comparisons of variables between multiple groups were performed using one-way ANOVA. Calculations were performed with SPSS (ver. 16.0, Chicago), and $P \leq 0.05$ was considered statistically significant.

\section{Results}

\section{Animal survival}

Four mice in the MI group and 3 mice in the iPS-CM group died during the 4 weeks of observation after cell transplantation. There was no significant difference in mortality rate between these two groups (Fig. 1, 4/12, 33\% vs. $3 / 12,25 \%, P>0.1$ ). We also found that among the total number of deaths, nearly $86 \%$ of mice died in the first week after MI (6/7). At the end of the experiment, there were at least 8 surviving animals in each group.

\section{Immunohistochemistry of single human iPS-CMs}

Flow cytometry was used to analyse cardiac troponin $\mathrm{T}$ in iPS-CMs. The results showed that the viability of cells in all batches was greater than $90 \%$ before transplantation, and the quality of differentiated cells was consistent among each batch. The identity of the CMs was verified by immunostaining for green fluorescent protein (GFP, green colour) and troponin $\mathrm{T}$ (red colour). The results from immunostaining and flow cytometry demonstrated that $85 \%$ of the total cells in the culture medium were iPS-CMs (Fig. 2b). $\beta$-Galactosidase staining did not show significant cellular senescence (blue colour, Fig. 2c).

\section{Cardiac function improvement after transplantation}

Heart function was assessed by a series of echocardiographic measurements collected on the 7th, 14th and 
A
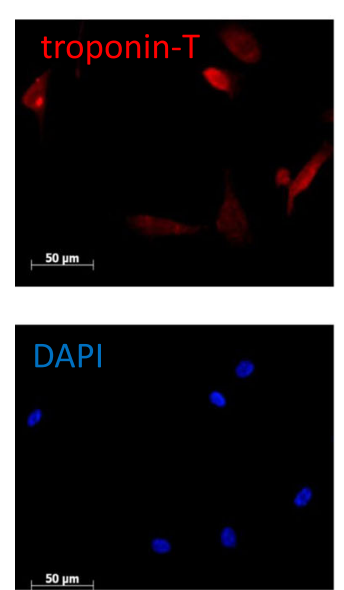
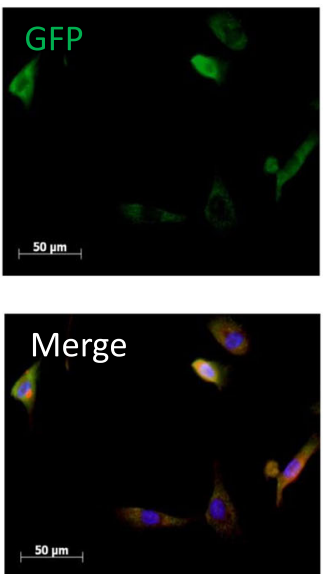

B

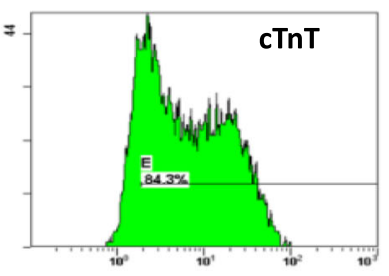

C

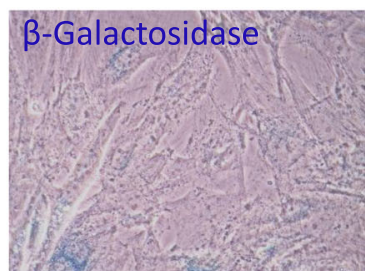

Fig. 2 Characterisation of iPS-CMs via the expression of the cardiac special marker troponin T. a Cardiac cells were stained for troponin T with red, GFP with green, and DAPI with blue; a merged image is also shown. $\mathbf{b}$ Flow cytometry analysis of cell viability using anti-cardiac troponin T. The results showed that the percentage of iPS-CMs was approximately $85 \%$. c $\beta$-Galactosidase staining did not show significant changes in cellular senescence

28th days following transplantation. On day 28, LVEF was significantly enhanced in the iPS-CMs group $(54.7 \pm 6.42 \%, n=9)$ compared with the MI group $(43.22 \pm 7.71 \%, n=8, \quad P<0.05$; Fig. 3a); LVIDs and LVIDd values in the iPS-CMs group $(4.32 \pm 0.71 \mathrm{~mm}$ and $3.21 \pm 0.72 \mathrm{~mm}$ ) were significantly lower than those in the MI group $(4.81+0.61 \mathrm{~mm}$ and $3.83+0.58 \mathrm{~mm}$, $P<0.05$; Fig. $3 b)$. We did not observe any significant alterations in LVEF, LVIDs or LVIDd values between these two groups on the 7th and 14th days post MI. Invasive haemodynamic measurement at 4 weeks after cell transplantation revealed that iPS transplantation improved both $+\mathrm{dP} / \mathrm{dt}(3638.9 \pm 507.3)$ and $-\mathrm{dP} / \mathrm{dt}$ $(-3415.5 \pm 526.1)$ in MI $(2657.64 \pm 426.25$ and $2283.47 \pm 338.73, P<0.05$, Fig. $3 c$, d).

\section{Cellular senescence evaluation}

Compared to their levels in the control animals, the animals subjected to the MI operation exhibited significantly elevated PAI-1, MMP-3 and IL-6 expression at the transcriptional level, which was attenuated by iPS transplantation. The mRNA levels of PAI-1, MMP-3 and IL-6 in the iPS-CM group were identical to those in the normal control group (Fig. 4).

\section{Immunohistochemical assessment of the infarct area}

Tissue sections were prepared and processed by Masson's trichrome staining 4 weeks after transplantation (Fig. 5). The iPS-CM group had a smaller infarcted area than the MI group $\left(2.57 \pm 0.52 \mathrm{~mm}^{2}\right.$ vs. $1.7 \pm 0.48 \mathrm{~mm}^{2}$, $P<0.05)$. We did not observe any excessive proliferation of transplanted iPS at the injection areas or other positions throughout the myocardial tissue.
iPS-CM engraftment and survival after transplantation Transplanted cells were detected by immunostaining for GFP and troponin $T$ in tissue sections at the end of the 4th week after transplantation (Fig. 6). Less than $1 \%$ of cells in the myocardial tissues survived after 4 weeks of transplantation, as shown by quantification of GFPpositive cells, which were mainly observed in the infarct border area of animals in the cell group. The number of GFP-positive cells at this point was drastically decreased compared with day 7 (13\%).

\section{Angiogenesis and decrease in apoptosis after transplantation}

The capillary density in the cell group $(156.7 \pm 42.3$ / $\mathrm{mm}^{2}$ ) was significantly higher than it was in the MI group $\left(44.5 \pm 13.4 / \mathrm{mm}^{2}\right)$, as detected by vWF staining $(P<0.05$, Fig. 7). Furthermore, TUNEL staining revealed a significantly lower percentage of apoptotic cells at the infarct border area in the cell transplantation group $(21.93 \pm 5.36 \%)$ than in the MI group $(41.63 \pm 8.29 \%$, $P<0.05$, Fig. 8).

\section{Discussion}

There are two major types of stem cells that have the potential for heart regeneration: multipotent stem cells (adult stem cells) and pluripotent stem cells (ESCs, embryonic stem cells or iPSs). However, both types of cells present high risks of teratoma formation and heterotopic differentiation after transplantation into the host's heart $[9,10]$. To avoid this problem, differentiated cells, such as stem cell-derived cardiomyocytes, are preferred in stem cell therapy. Recent preclinical [11-13] and clinical studies [14] have demonstrated the great beneficial effect of human ESC-derived cardiomyocytes (ESC-CMs) and iPS-CMs in LV remodelling. Furthermore, iPS-CMs are 


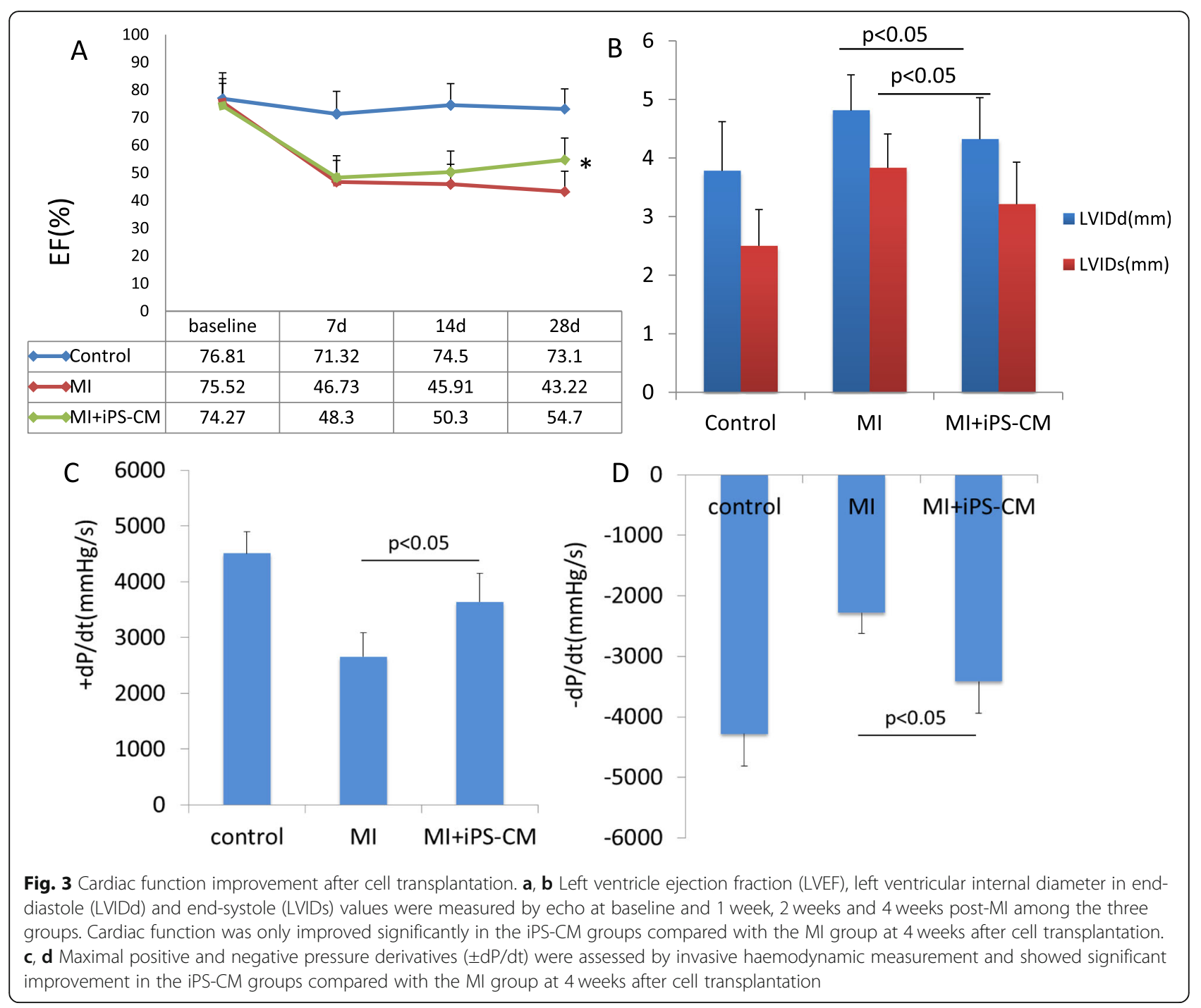

autologous cells that are generated from somatic cells. Thus, there are fewer ethical concerns and a decreased need for immunosuppression after transplantation related to treatment with iPS-CMs compared with ESCCMs. iPS-CMs should provide more predictable therapeutic effects and abilities for "off-the-shelf" use. However, the mechanism by which iPS-CMs improve heart function is not clear. In this paper, we found that transplantation of iPS-CMs produced therapeutic benefits that improved heart function and reduced infarct size in a mouse MI model. This effect was associated with angiogenesis and suppressed apoptosis of native cardiomyocytes in ischaemic cardiac tissue, which indicated that the mechanism of functional improvement was mediated by
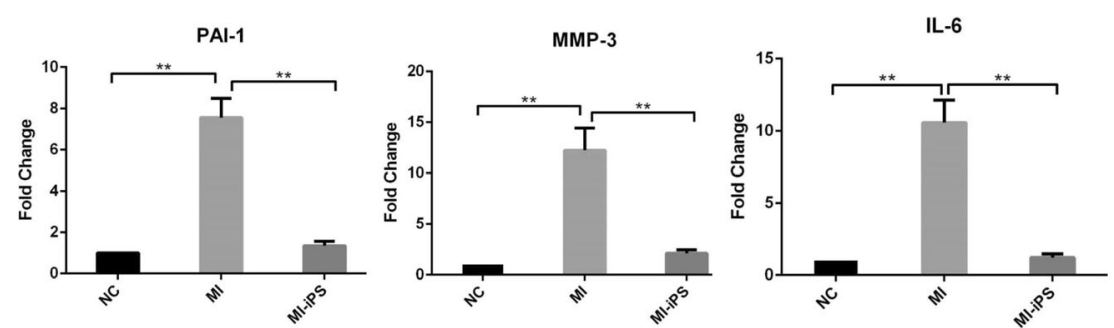

Fig. 4 Cellular senescence improvement after cell transplantation. RT-PCR showed that the mRNA levels of PAI-1, MMP-3 and IL-6 were significantly increased in the Ml group compared with the control group, and they significantly decreased after iPS-CM transplantation 


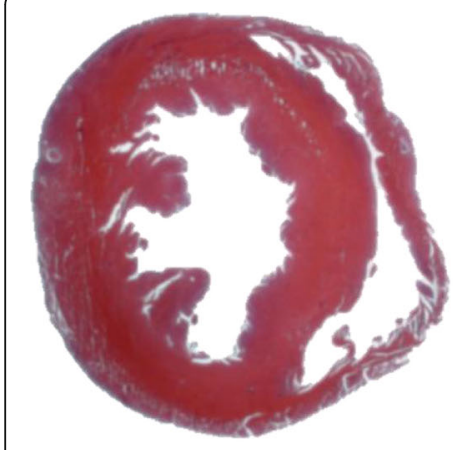

Control

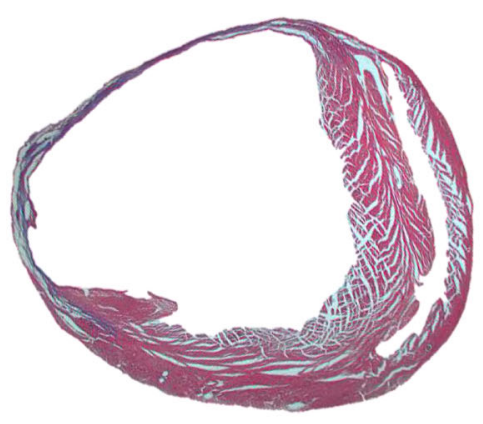

$\mathrm{MI}$

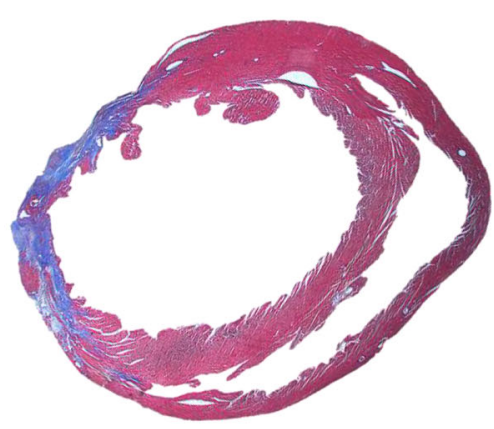

MI+iPSC-CM

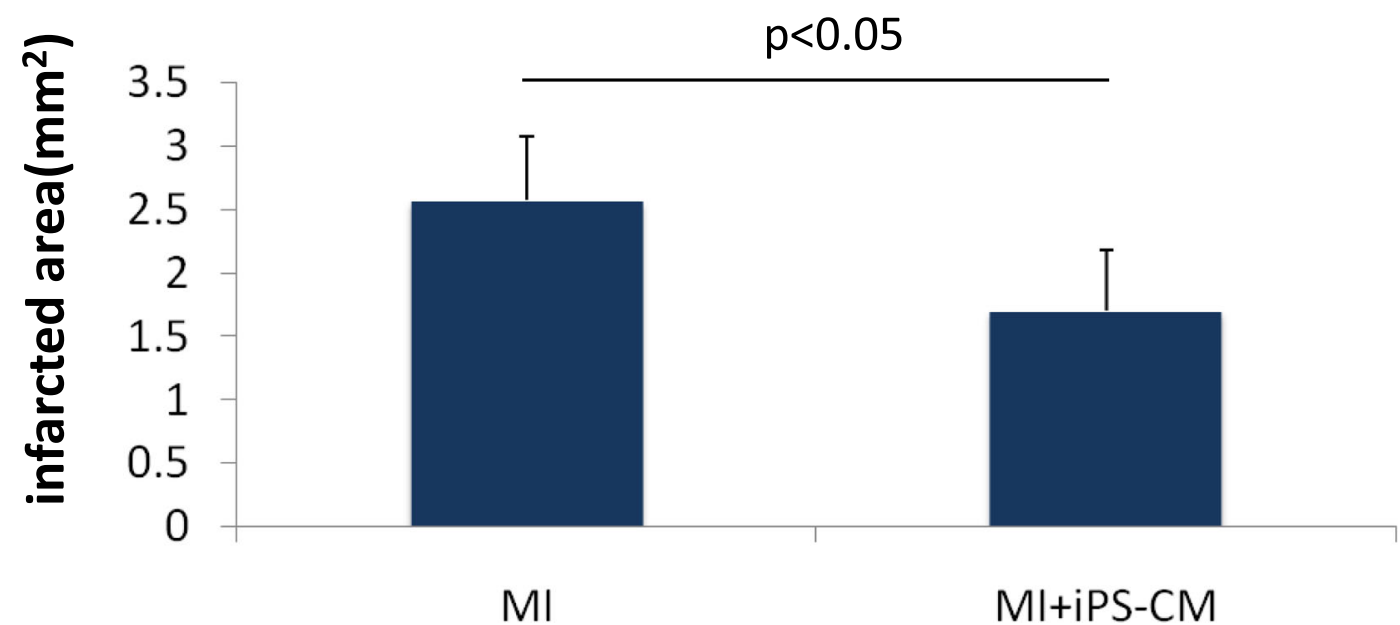

Fig. 5 Infarct size reduction 4 weeks after cell transplantation. Masson trichrome staining showed that the infarct area (blue colour, $P<0.05)$ in the iPS-CM group was significantly smaller than it was in the MI group

paracrine effects. These data have important clinical implications for the development of iPS-based therapies.

In this study, purified human iPS-CMs (> 85\% pure in culture medium) were injected into the myocardium directly after MI. Four weeks after transplantation, the $\mathrm{EF}$ and $\mathrm{dP} / \mathrm{dt}$ of the iPS-CM group improved significantly and there was a smaller infarct area than there was in the control group. Cell engraftment after transplantation was identified by GFP immunostaining. A small number of cells $(<1 \%)$ could be observed on the 4th week after transplantation into myocardium tissues. Therefore, it is unlikely that the transplanted cells directly contributed to the improvement of cardiac function. However, we found a significant increase in capillary density and a reduction in native $\mathrm{CM}$ apoptosis in the heart tissues following injection of cells. The transplanted cells enhanced angiogenesis and preserved the survival rate of the surrounding residential CMs via paracrine effects, which may ultimately lead to functional improvement. Furthermore, we did not observe any teratomas on any region of the heart.

Despite the great potential of iPSCs in regenerative medicine and cell therapy, there are several major hurdles that need to be addressed prior to their clinical application. First, the protocol using viral vectors to produce iPSCs may result in mutagenesis or malignant transformation. It is important to develop methods for reprogramming iPSCs without the need for any genetic modification and to obtain high purity (100\%) and a high volume (10-100 billion) of functional cardiomyocytes prior to clinical application; such methods could involve single small molecules or virus-free methods $[15,16]$. Second, safety evaluations, including arrhythmogenesis and tumorigenesis, are important concerns in cell-based therapy. Existing efforts mostly focus on cardiac differentiation, and limited attention has been paid to the important fact that transplanted CMs also need to exhibit a mature phenotype similar to that of 

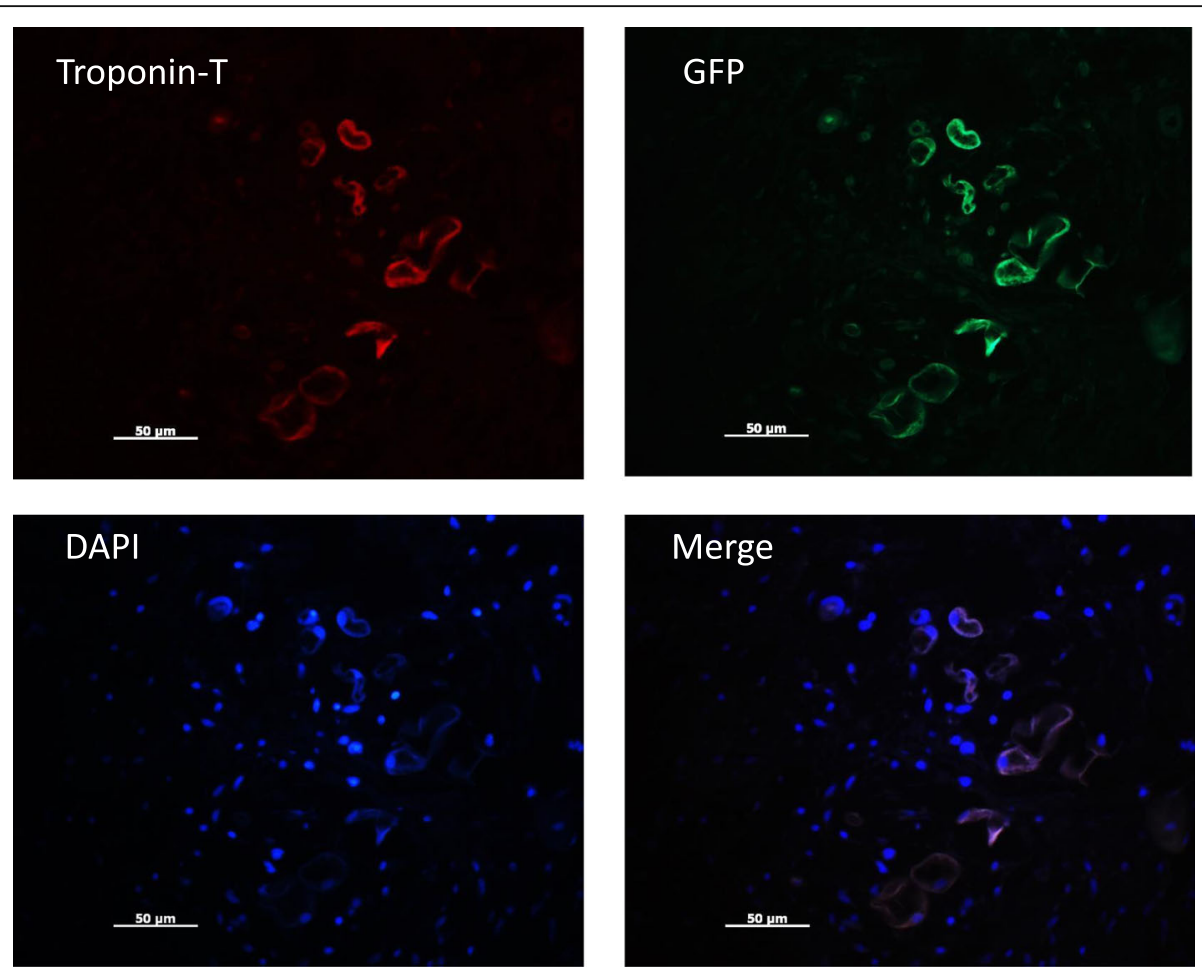

Fig. 6 iPS-CM engraftment and survival after cell transplantation. Surviving transplanted cells were stained with troponin T (red), GFP (green), and DAPI (blue) at the infarct border area 4 weeks after transplantation; a merged image is also shown

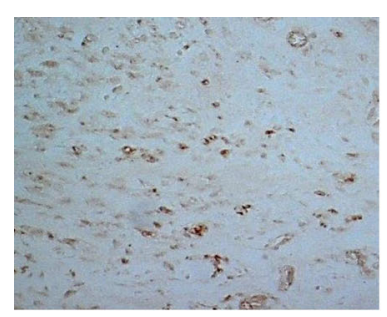

Control

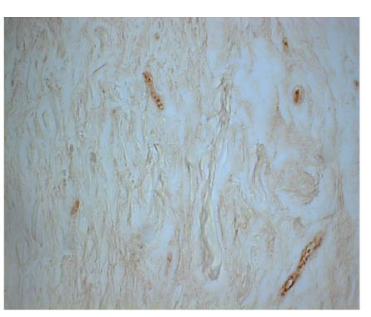

MI

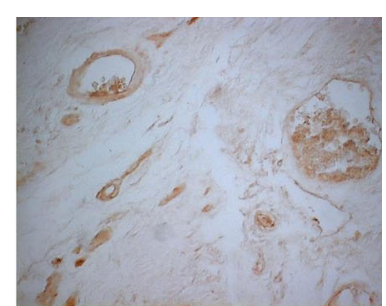

Ml+iPS-CMs

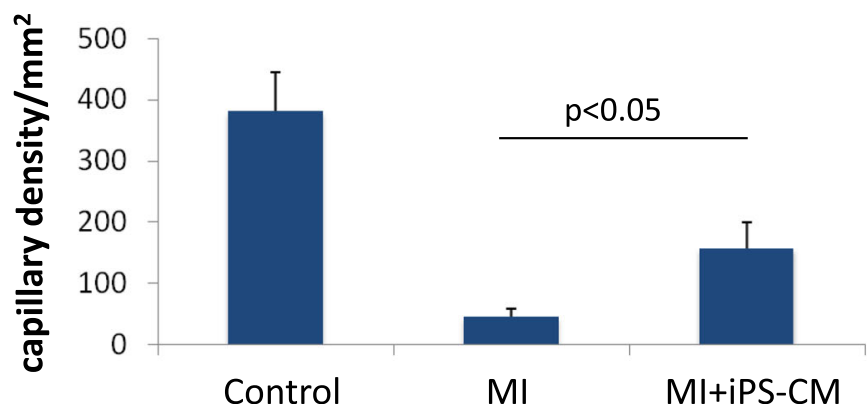

Fig. 7 Angiogenesis after cell transplantation. Anti-von Willebrand factor (VWF) staining showed that the capillary density (brown colour) at the infarct border area was significantly higher in the iPS-CM group than it was in the Ml group 4 weeks after transplantation 


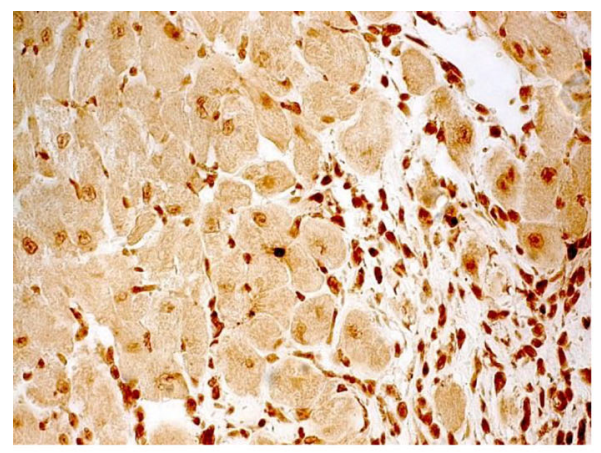

$\mathrm{MI}$

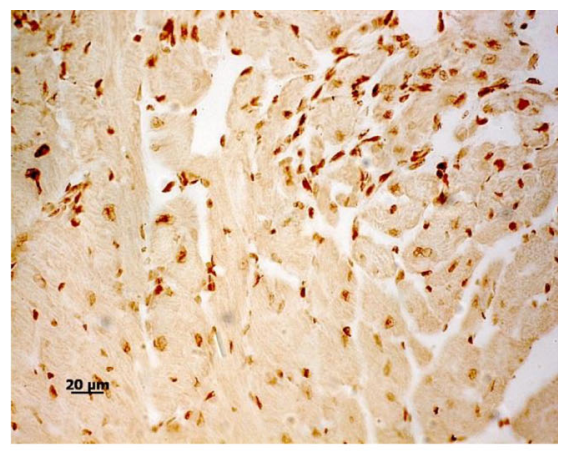

MI+iPS-CMs

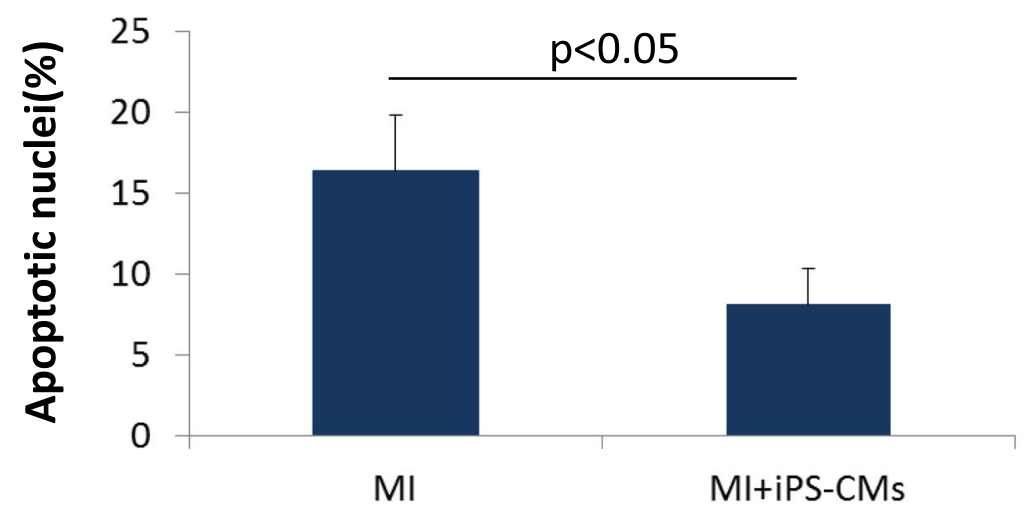

Fig. 8 Anti-apoptosis after cell transplantation. Terminal deoxynucleotidyl nick end-labelling (TUNEL) staining revealed that there was a lower percentage of apoptotic cells at the infarct border area in the iPS-CM group than there was in the MI group 4 weeks after transplantation

the host myocardium. The immature electrical phenotypes of these cells might contribute to proarrhythmias. Proarrhythmic effects have been reported in ESC-CM graft studies [11, 17]. iPS-CMs also exhibit immature electrophysiological properties compared with mature adult CMs $[18,19]$. However, the risk of arrhythmias following hESC-CM transplantation in injured hearts has not been determined. Future studies are needed to improve the maturation of these iPS$\mathrm{CMs}$ before transplantation. Tumorigenesis is also an important concern in cell type choice. In this study, we did not observe any teratoma formation because CMs are a terminal stage of iPS and have limited ability to proliferate. Multilineage-differentiating stress enduring (Muse) cells are nontumorigenic endogenous pluripotent-like stem cells that can be obtained from various tissues, including the bone marrow, and they have shown long-lasting tissue repair and functional recovery after acute myocardial infarction in a rabbit model [20]. This may provide another selectable safe cell type for MI treatment.

Third, the mechanism by which cell transplantation improves cardiac function improvement is still not clear. The initial promise was that these transplanted stem cells could directly contribute to heart regeneration. However, recent studies proved that most of their beneficial effects are attributed to indirect actions, including paracrine actions, and modulation of the extracellular matrix and apoptosis, which is very similar to the results of this study. Unfortunately, which factors or proteins are the key contributors to the improvement of cardiac function has not yet been determined. Finally, one of the urgent problems of cell therapy is the low survival rate and engraftment after transplantation [21]. A recent study using human iPS-CMs for transplantation in a mini-pig MI model showed that only a small number of iPS-CMs were detected by fluorescence in situ hybridization at the 8th week after transplantation, indicating poor long-term survival and engraftment of the cells [22]. This progressive loss of transplanted cells could be a result of allogeneic immune rejection after transplantation. As an alternative to direct cell replacement, the release of exosomes from iPSC-CMs represents a new type of iPSC-based cardiomyogenesis therapy for heart regeneration [23]. These exosomes are known to possess anti-apoptotic effects, stimulate angiogenesis, reduce infarct size, and improve cardiac recovery. Positive outcomes of this study are associated with the secretion of paracrine factors in the 
absence of adequate cell survival, which may be mediated by the release of exosomes. Compared with those derived from iPSC-CMs alone, exosomes derived from MSCs cocultured with iPSC-CMs promoted longer survival and enhanced therapeutic effects in cells after transplantation [24]. The beneficial effects of exosomes secreted by iPSCCMs in cardiac regeneration should be clarified in future studies.

This study provides support for the application of iPS$\mathrm{CM}$ therapy for heart regeneration. We clarified the mechanism of heart function improvement after cell transplantation, which is a paracrine effect. Strategies to improve maturation, cell survival and therapeutic efficacy after transplantation require further investigation.

\section{Conclusions}

Our results demonstrate that transplantation of human iPS-CMs can improve heart function via their paracrine effect in a mouse model of myocardial infarction.

\section{Study limitations and future perspectives}

There are several limitations in this study. First, allogeneic murine model will induce immunoreaction after human cell transplantation, cell loss during the injection and immunorejection might have affected the experimental outcomes. The arrhythmogenesis after cell transplantation cannot be evaluated due to the high intrinsic beating rate and small size of the mouse heart. Future study using a large animal transplantation model receiving immunosuppression should allow better resolution for detailed functional and safety assessments. The optimal strategies of cardiac tissue bioengineering to improve cell survival and therapeutic effects also remain to be addressed.

\section{Abbreviations \\ iPS: Induced pluripotent stem cells; MI: Myocardial infarction; HF: Heart failure; iPS-CMs: Induced pluripotent stem cell-derived cardiomyocytes; LV: Left ventricle; LVEF: Left ventricle ejection fraction; LVIDd: Left ventricular internal diameter in end-diastole; LVIDs: Left ventricular internal diameter in end-systole; ESC: Embryonic stem cells; GFP: Green fluorescent protein; ICR: Imprinting Control Region; LAD: Left anterior descending; LVPW: Left ventricular posterior wall thickness; IVS: Intraventricular septum thickness; LVEDP: LV end-diastolic pressure; LVESP: LV end-systolic pressure}

\section{Acknowledgements}

Thanks to Zhaowen Xiao for his help in the progress of the experiment. Although not listed in the author's list, his efforts are equally important to us. Thanks to Yanru Yang for her help in the publication process of this paper.

\section{Authors' contributions}

JX participated in the performance of the research, in research design and in the writing of the paper. YZY collected the samples, performed the additional experiments and analysed the data. DM designed the study and drafted the manuscript. All authors read and approved the final manuscript.

\section{Funding}

This work was supported by grant from Basic Research Foundation of Shenzhen (No: JCYJ 20130402101926972), National Natural Science Foundation of China (No: 81202529) and China Postdoctoral Science Foundation (No:2015 M582416.)

\section{Availability of data and materials}

The data that support the findings of this study are available from the corresponding author upon reasonable request.

\section{Ethics approval and consent to participate}

All animal experimental procedures were approved by the Committee on the Use of Live Animals in Teaching and Research at the University of Shenzhen. All ethical procedures conformed to the principles of 1964 Declaration of Helsinki and its latest 2008 amendments. The treatment of experimental animals is also in strict accordance with the requirements of the laboratory animal management centre.

\section{Consent for publication}

Not applicable.

\section{Competing interests}

The authors declare that they have no competing interests.

\section{Author details}

${ }^{1}$ Department of Geriatrics, The Second Clinical Medical College of Jinan University, Shenzhen 518020, Guangdong, China. Bioisland Laboratory, Biomedical Equipment Department, Building 3, No.188 KaiYuan Road, Huangpu District, Guangzhou, Guangdong, China.

Received: 7 April 2020 Revised: 30 June 2020

Accepted: 3 July 2020 Published online: 17 July 2020

\section{References}

1. Zhang J, Wilson GF, Soerens AG, Koonce CH, Yu J, Palecek SP, et al. Functional cardiomyocytes derived from human induced pluripotent stem cells. Circ Res. 2009:104(4):e30-41.

2. Yu T, Miyagawa S, Miki K, Saito A, Fukushima S, Higuchi T, et al. In vivo differentiation of induced pluripotent stem cell-derived cardiomyocytes. Circ J. 2013;77(5):1297-306

3. Carpenter L, Carr C, Yang CT, Stuckey DJ, Clarke K, Watt SM. Efficient differentiation of human induced pluripotent stem cells generates cardiac cells that provide protection following myocardial infarction in the rat. Stem Cells Dev. 2011;21(6):977-86.

4. Kawamura M, Miyagawa S, Miki K, Saito A, Fukushima S, Higuchi T, et al. Feasibility, safety, and therapeutic efficacy of human induced pluripotent stem cell-derived cardiomyocyte sheets in a porcine ischemic cardiomyopathy model. Circulation. 2012;126(11 SUPPL.1):29-37.

5. Ye L, Chang Y-H, Xiong Q, Zhang P, Zhang L, Somasundaram P, et al. Cardiac repair in a porcine model of acute myocardial infarction with human induced pluripotent stem cell-derived cardiovascular cell populations. Cell Stem Cell. 2014;15(6):750-61.

6. Higuchi T, Miyagawa S, Pearson JT, Fukushima S, Saito A, Tsuchimochi H, et al. Functional and electrical integration of induced pluripotent stem cellderived cardiomyocytes in a myocardial infarction rat heart. Cell Transplant. 2015:24(12):2479-89.

7. van Laake LW, Passier R, Monshouwer-Kloots J, Verkleij AJ, LiPS DJ, Freund C, et al. Human embryonic stem cell-derived cardiomyocytes survive and mature in the mouse heart and transiently improve function after myocardial infarction. Stem Cell Res. 2007;1(1):9-24.

8. Sahn DJ, DeMaria A, Kisslo JA, Weyman AE. Recommendations regarding quantitation in M-mode echocardiography: results of a survey of echocardiographic measurements. Circulation. 1978;58(6):1072-83.

9. Ahmed RP, Ashraf M, Buccini S, Shujia J, Haider HK. Cardiac tumorgenic potential of induced pluripotent stem cells in an immunocompetent host with myocardial infarction. Regen Med. 2011;6(2):171-8.

10. Zhang Y, Wang D, Chen M, Yang B, Zhang F, Cao K. Intramyocardial transplantation of undifferentiated rat induced pluripotent stem cells causes tumorigenesis in the heart. PLoS One. 2011;6(4):2-7.

11. Chong JJ, Yang X, Don CW, et al. Human embryonic-stem-cell-derived cardiomyocytes regenerate non-human primate hearts. Nature. 2014; 510:273-7

12. Shiba Y, Gomibuchi T, Seto T, et al. Allogeneic transplantation of iPS cell-derived cardiomyocytes regenerates primate hearts. Nature. 2016; 538:388-91. 
13. Liu Y-W, Chen B, Yang X, et al. Human ESC-derived cardiomyocytes restore function in infarcted hearts of non-human primates. Nat Biotechnol. 2018; 36(7):597-605.

14. Menasche P, Vanneaux V, Fabreguettes JR, et al. Towards a clinical use of human embryonic stem cell-derived cardiac progenitors: a translational experience. Eur Heart J. 2015;36:743-50.

15. Pasha Z, Haider HK, Ashraf M. Efficient non-viral reprogramming of myoblasts to stemness with a single small molecule to generate cardiac progenitor cells. PLoS One. 2011;6(8):e23667.

16. Woltjen K, Paca A, Mohseni P, Norrby K, Mileikovsky M, Kaji K. Virus-free induction of pluripotency and subsequent excision of reprogramming factors. Nature. 2009;458(7239):771-5.

17. Liao SY, Liu Y, Siu CW, Zhang Y, Lai WH, Au KW, et al. Proarrhythmic risk of embryonic stem cellderived cardiomyocyte transplantation in infarcted myocardium. Hear Rhythm. 2010;7(12):1852-9. https://doi.org/10.1016/j. hrthm.2010.09.006.

18. Ronaldson-Bouchard K, Ma SP, Yeager K, Chen T, Song L, Sirabella D, Morikawa K, Teles D, Yazawa M, Vunjak-Novakovic G. Advanced maturation of human cardiac tissue grown from pluripotent stem cells. Nature. 2018; 556:239-43.

19. Ribeiro AJ, Ang YS, Fu JD, Rivas RN, Mohamed TM, Higgs GC, Srivastava D, Pruitt BL. Contractility of single cardiomyocytes differentiated from pluripotent stem cells depends on physiological shape and substrate stiffness. Proc Natl Acad Sci U S A. 2015;112:12705-10.

20. Yamada Y, Wakao S, Kushida Y, Minatoguchi S, Mikami A, Higashi K, Baba S, Shigemoto T, Kuroda Y, Kanamori H, Amin M, Kawasaki M, Nishigaki K, Taoka M, Isobe T, Muramatsu C, Dezawa M, Minatoguchi S. S1P-S1PR2 axis mediates homing of muse cells into damaged heart for long-lasting tissue repair and functional recovery after acute myocardial infarction. Circ Res. 2018;122(8):1069-83.

21. Martens A, Baraki SVRH, Rathert C, Schecker N, Zweigerdt R, Schwanke K, et al. Substantial early loss of induced pluripotent stem cells following transplantation in myocardial infarction. Artif Organs. 2014;38(11):978-84.

22. Kawamura M, Miyagawa S, Fukushima S, Saito A, Miki K, Ito E, et al. Enhanced survival of transplanted human induced pluripotent stem cellderived cardiomyocytes by the combination of cell sheets with the pedicled omental flap technique in a porcine heart. Circulation. 2013; 128(SUPPL.1):87-94.

23. Ong SG, Lee WH, Zhou Y, Wu JC. Mining exosomal microRNAs from human-induced pluripotent stem cells-derived cardiomyocytes for cardiac regeneration. Methods Mol Biol. 2018;1733:127-36.

24. Yoshida S, Miyagawa S, Fukushima S, Kawamura T, Kashiyama N, Ohashi F, Toyofuku T, Toda K, Sawa Y. Maturation of human induced pluripotent stem cell-derived cardiomyocytes by soluble factors from human mesenchymal stem cells. Mol Ther. 2018;26(11):2681-95.

\section{Publisher's Note}

Springer Nature remains neutral with regard to jurisdictional claims in published maps and institutional affiliations.

Ready to submit your research? Choose BMC and benefit from:

- fast, convenient online submission

- thorough peer review by experienced researchers in your field

- rapid publication on acceptance

- support for research data, including large and complex data types

- gold Open Access which fosters wider collaboration and increased citations

- maximum visibility for your research: over $100 \mathrm{M}$ website views per year

At $\mathrm{BMC}$, research is always in progress.

Learn more biomedcentral.com/submissions 